\title{
Reduced Staphylococcus aureus biofilm formation in the presence of chitosan-coated iron oxide nanoparticles
}

This article was published in the following Dove Press journal:

International Journal of Nanomedicine

7 December 2016

Number of times this article has been viewed

\author{
Si-feng Shi ${ }^{1,2}$ \\ Jing-fu Jia ${ }^{3}$ \\ Xiao-kui Guo ${ }^{4}$ \\ Ya-ping Zhao ${ }^{3}$ \\ De-sheng Chen ${ }^{2}$ \\ Yong-yuan Guo 2 \\ Xian-long Zhang ${ }^{2}$
}

'Department of Orthopedics, the Affiliated Hospital of Xu Zhou

Medical University, Xu Zhou, Jiangsu,

${ }^{2}$ Department of Orthopedic Surgery,

Shanghai sixth People's Hospital,

Shanghai Jiao Tong University,

Shanghai, ${ }^{3} \mathrm{School}$ of Chemistry

and Chemical Technology, Shanghai

Jiao Tong University, Shanghai,

${ }^{4}$ Department of Medical Microbiology and Parasitology, Institutes of Medical Sciences, Shanghai Jiao Tong University School of Medicine, Shanghai, People's Republic of China
Correspondence: Si-feng Shi

Department of Orthopedics, the

Affiliated Hospital of XuZhou Medical

University, XuZhou, No 99, West Huaihai

Road, XuZhou, Jiangsu 22 I000, People's

Email shisifeng2007।@I63.com

Xian-long Zhang

Department of Orthopaedic Surgery,

Shanghai Sixth People's Hospital,

Shanghai Jiao Tong University,

No 600 Yi Shan Road, Xu Hui

District, Shanghai, People's

Republic of China

$\mathrm{Tel}+862$ I 6436 9|8I

Email zhangxianlong2010I@I63.com
Republic of China

Abstract: Staphylococcus aureus can adhere to most foreign materials and form biofilm on the surface of medical devices. Biofilm infections are difficult to resolve. The goal of this in vitro study was to explore the use of chitosan-coated nanoparticles to prevent biofilm formation. For this purpose, S. aureus was seeded in 96-well plates to incubate with chitosan-coated iron oxide nanoparticles in order to study the efficiency of biofilm formation inhibition. The biofilm bacteria count was determined using the spread plate method; biomass formation was measured using the crystal violet staining method. Confocal laser scanning microscopy and scanning electron microscopy were used to study the biofilm formation. The results showed decreased viable bacteria numbers and biomass formation when incubated with chitosan-coated iron oxide nanoparticles at all test concentrations. Confocal laser scanning microscopy showed increased dead bacteria and thinner biofilm when incubated with nanoparticles at a concentration of $500 \mu \mathrm{g} / \mathrm{mL}$. Scanning electron microscopy revealed that chitosan-coated iron oxide nanoparticles inhibited biofilm formation in polystyrene plates. Future studies should be performed to study these nanoparticles for anti-infective use.

Keywords: chitosan-coated iron oxide nanoparticles, Staphylococcus aureus, biofilm

\section{Introduction}

Staphylococcus aureus, a Gram-positive pathogen, is a ubiquitous skin microflora and community-acquired pathogen. It can opportunistically cause various chronic infections in the medical field. ${ }^{1,2} S$. aureus is one of the most frequently isolated pathogens in wound infections and osteomyelitis. ${ }^{1}$ It is reported that $S$. aureus caused almost $80 \%$ of osteomyelitis in 2011. ${ }^{3}$ Methicillin-resistant $S$. aureus infection is especially difficult to eradicate. With the increasing number of indwelling medical devices used in treating bone disease, foreign body device infections have become increasingly prevalent in the orthopedics field. ${ }^{4} S$. aureus can adhere to most of these foreign materials and form biofilm on the surface of medical devices. Once established, such foreign body related infections are difficult to resolve.

Biofilms are a group of microorganisms growing collectively in a self-produced hydrated polymeric matrix on surfaces and interfaces. ${ }^{5}$ The importance of biofilms in the process of chronic infections has been widely recognized over the past two decades, especially in medical implants related infection. ${ }^{1}$ The ability of formation of biofilm by $S$. aureus is closely associated with its capacity to produce an extracellular mucous substance, polysaccharide intercellular adhesion (PIA). ${ }^{6}$ The main component of PIA is glycosaminoglycans. ${ }^{7}$ PIA provides the biofilm with a stable structure, helps the bacteria to adhere to material surfaces, and protects the bacteria embedded in it from 
being killed by the immune system or antibiotics. ${ }^{8}$ Biofilm allows the bacteria to survive in hostile environmental conditions against various environmental stresses, including antibiotic treatment. ${ }^{9}$ Reduced antibiotic susceptibility of biofilm bacteria compared with planktonic cultures make biofilm-associated infections extremely difficult to treat. ${ }^{8}$ In addition, these biofilms provide a barrier that reduces penetration of antimicrobial agents. Treatment of biofilm-related infections requires high doses and long-term use of antibiotics and the resultant inactivation is not always satisfactory. ${ }^{7}$ Low concentrations of antibiotics have even been reported to enhance the growth of biofilms..$^{8,10,11}$ The chronic infection usually cannot be eradicated unless the implant is removed after various treatments, and this procedure places a large burden on patients and the medical officials. ${ }^{12}$

So, novel and innovative approaches are needed for the prevention of biofilm formation and the treatment of formed biofilm related infectious diseases. ${ }^{6}$ Recent progress in nanotechnology provides a new approach for treatment of these diseases. Several nanoparticles with antibacterial capacity have been studied. ${ }^{5,13,14}$ Chitosan nanoparticles could inhibit the growth of various bacteria. Exposure of Salmonella choleraesuis to chitosan nanoparticles led to the disruption of cell membranes and the leakage of cytoplasm. ${ }^{15,16}$ In order to allow the implanted material to have antibacterial properties, coating and impregnation of these antibacterial nanomaterials has also been studied. ${ }^{5}$

Iron oxide nanoparticles have also been studied in recent years. Chatterjee et $\mathrm{al}^{17}$ showed that nanoparticles of iron oxide have an inhibitory effect on Escherichia coli in a concentration dependent manner. The magnetic property of the iron oxide nanoparticles make them more promising in the targeted therapy of infectious disease. When an external magnetic field is added, the nanoparticles can be inducted to the local area of infection. Moreover, iron oxide nanoparticles have shown some extent of antibacterial effect in several studies. ${ }^{18,19}$

Chitosan has good biocompatibility and has been approved for use by the United States Food and Drug Administration as a drug carrier and bioengineering material. In our previous studies, we coated chitosan onto iron oxide nanoparticles to gain good biocompability to human osteoblast cells. ${ }^{20}$ The present study was undertaken to investigate the potency of chitosan-coated iron oxide nanoparticles to prevent biofilm formation against $S$. aureus which is the main organism in orthopedic implant infections. ${ }^{21}$ The results demonstrated that chitosan-coated iron oxide nanoparticles inhibit biofilm formation by $S$. aureus by inhibiting the formation of biofilm biomass and decreasing the number of live bacteria.

\section{Materials and methods Preparation of the nanoparticles}

The nanoparticles were synthesized according to the previous method..$^{20,22}$ Briefly, ferric chloride was added to ferrous sulphate solution to form a mixed solution. Dissolved oxygen was removed. Ammonia was then added and constant stirring was performed for 30 minutes until precipitate appeared. After washing four times with deionized water, chitosan (the degree of deacetylation was $85 \%$ and the average molecular weight was $220 \mathrm{kDa}$ ) was added to the solution. The $\mathrm{pH}$ value was mediated to $\mathrm{pH} 4.0$ using phosphoric acid. Then constant stirring was performed for 12 hours. The nanoparticles suspended in the liquid were separated with a magnet and sterilized for further use. Transmission electron microscopy, $\mathrm{X}$-ray diffraction, and vibrating sample magnetometry were employed to characterize the nanoparticles. ${ }^{20}$ Transmission electron microscopy showed that the nanoparticles were $15-25 \mathrm{~nm}$ in diameter, with sphere-like shape and positive surface charge, and the characterization has been reported in our previous studies. ${ }^{20}$

\section{Bacterial strains and culture conditions}

A regularly used standard laboratory strain (25923; American Type Culture Collection [ATCC], Manassas, VA, USA) was chosen in the study of chitosan-coated iron oxide nanoparticles' effect in preventing biofilm formation. The bacteria were plated on a lysogeny broth (LB) agar plate. A single colony of the bacteria in the plate was selected and inoculated into centrifuge tubes containing $4 \mathrm{~mL}$ of LB broth. S. aureus inoculated in the medium were grown at $37^{\circ} \mathrm{C}$ for 16 hours under agitation at $200 \mathrm{rpm}$ (Orbital shaker model 4520; Forma Scientific Inc, Marietta, OH, USA).

\section{Static biofilm formation assay on polystyrene culture plates}

For biofilm preparation, $S$. aureus cells at a concentration of $5 \times 10^{8}$ colony-forming units $(\mathrm{CFU}) / \mathrm{mL}$ were seeded in a 96-well microplate (sterilized; Corning Incorporated, Corning, NY, USA). LB supplemented with chitosan-coated iron oxide nanoparticles were added to each well at different concentrations ( $200 \mu \mathrm{g} / \mathrm{mL}, 500 \mu \mathrm{g} / \mathrm{mL}, 1 \mathrm{mg} / \mathrm{mL}, 2 \mathrm{mg} / \mathrm{mL}$, $4 \mathrm{mg} / \mathrm{mL}$ ). Chitosan nanoparticles were used as a control. After covering with a lid, the microplate containing $S$. aureus cells was incubated for 48 hours without shaking at $37^{\circ} \mathrm{C}$.

\section{Biofilm bacteria counts}

The absolute number of bacteria cells present in the formed biofilms was determined using the spread plate method. ${ }^{23}$ The 
biofilm-forming cells in the microwell were rinsed three times with phosphate-buffered saline (PBS) to wash off planktonic bacteria. Biofilm bacterial cells were detached by exposition to a low energy sonication water bath (Xinzhi, Ning Bo, People's Republic of China) for 1 minute and centrifuged (Thermo Fisher Scientific, Waltham, MA, USA) at 4000 rpm for 5 minutes to form pellet cells. The biofilm cells were resuspended in $1 \mathrm{~mL}$ of LB and dispersed using homogenization as described above, and serial ten-fold dilutions were plated on LB agar plates for colony counts.

\section{Biofilm formation analysis with crystal violet staining}

The crystal violet (CV) staining method was also used to assess the effect of chitosan-coated magnetic nanoparticles on the inhibition of biofilm growth. ${ }^{714} \mathrm{~S}$. aureus cells at a concentration of $5 \times 10^{8} \mathrm{CFU} / \mathrm{mL}$ were inoculated into the wells of a $96-$ well microplate containing $100 \mu \mathrm{L}$ of liquid medium containing nanoparticles at various concentrations. The wells with chitosan nanoparticles and without nanoparticles were set as controls. After 1, 6, 12, and 24 hours of biofilm formation at a temperature of $37^{\circ} \mathrm{C}$, the plate wells were washed three times with PBS to remove planktonic cells. A $1 \%$ solution of $\mathrm{CV}$ was added to each well to stain the cells for 15 minutes. Then, the wells were rinsed thoroughly three times with water. Biofilm biomass was quantified by the addition of $100 \mu \mathrm{L}$ of $95 \%$ ethanol to each CV-stained microplate well, and the absorbance was determined with a plate reader (Bio-Tek, Winooski, VT, USA) at optical density (OD) $600 .{ }^{13}$ S. aureus without nanoparticles was set as $100 \%$. The relative percentage of biomass for the other samples was calculated relative to that of the control. All samples were incubated together in the same plate and under the same conditions.

\section{Confocal laser scanning microscopy (CLSM)}

CLSM was used to determine the effect of chitosan-coated iron oxide nanoparticles on the viability of the adherent biofilm. Biofilm viability was determined using a double live/ dead staining kit (BacLight Bacterial Viability Kits [Invitrogen Molecular Probes]; Life Technologies, Carlsbad, CA, USA) containing nucleic acid stains SYTO 9 and propidium iodide (PI). The biofilm formation assay described above was performed with minor changes. In brief, the polystyrene plates ( $1 \mathrm{~mm}$ thick and $5 \mathrm{~mm}$ in diameter) were placed in the bottom of the 12-well plate. The plate was inoculated with a freshly grown bacteria suspension and the nanoparticles were added to the suspension. After 48 hours of incubation, the polystyrene plates were gently removed, rinsed by immersing in PBS, leaving only the adhered bacteria and then stained using the live/dead BacLight kit according to the manufacturer's instructions. The biofilms were incubated in the SYTO9/PI staining mixture for 15 minutes. Viable bacteria with intact cell membranes were stained green, whereas dead bacteria with damaged membranes were stained red. Stained samples were visualized by CLSM (LSM 5 PASCAL; Carl Zeiss, Jena, Germany), with the following excitation/emission detectors and filter sets: for SYTO 9, 480/500 and for PI, 490/635.

\section{Biofilm prevention assay on polystyrene plates by scanning electron microscopy (SEM)}

The polystyrene plates ( $1 \mathrm{~mm}$ thick and $5 \mathrm{~mm}$ in diameter) were sterilized and placed into the wells of 12-well plates for biofilm formation. Aliquots $(1 \mathrm{~mL})$ of the bacteria cell suspensions containing chitosan-coated nanoparticles were seeded into each well. The polystyrene plates with cells grown in nanoparticle-free medium were utilized as a control. The plates were incubated at $37^{\circ} \mathrm{C}$ for 24 hours and then gently washed three times with PBS to remove non-adherent bacteria. The adherent bacteria were fixed and dehydrated. After being fixed with $2.5 \%$ glutaraldehyde for 3 hours at $4{ }^{\circ} \mathrm{C}$, the surfaces were rinsed three times and subsequently fixed with $0.1 \%$ osmium tetraoxide for 1 hour. The samples were dehydrated through a graded ethanol series $(50 \%, 70 \%$, $80 \%, 90 \%, 95 \%$, and $99.5 \%$ ) for 10 minutes each at room temperature. After critical-point drying and 1200 bar pressure at $40^{\circ} \mathrm{C}$, the samples were examined using SEM (XL-30 ESEM; Philips, Amsterdam, Netherlands). ${ }^{5,24}$

\section{Statistics}

All quantitative experiments were performed at least three times. Data were presented as mean values \pm standard deviation and analyzed using SPSS software (v 13.0; IBM Corporation, Armonk, NY, USA). Student's $t$-test was used for statistical analysis. $P<0.05$ was considered statistically significant.

\section{Results}

\section{The viable S. aureus counts in the biofilm}

The effectiveness of chitosan-coated iron oxide nanoparticles to inhibit bacterial colonization was examined. The nanoparticles with different concentrations were added to co-culture with the bacteria for 24 hours. As can be seen in Figure 1, chitosan-coated iron oxide nanoparticles were able to restrict $S$. aureus biofilm bacterial growth at all the experimental concentrations compared to the control and the restriction 


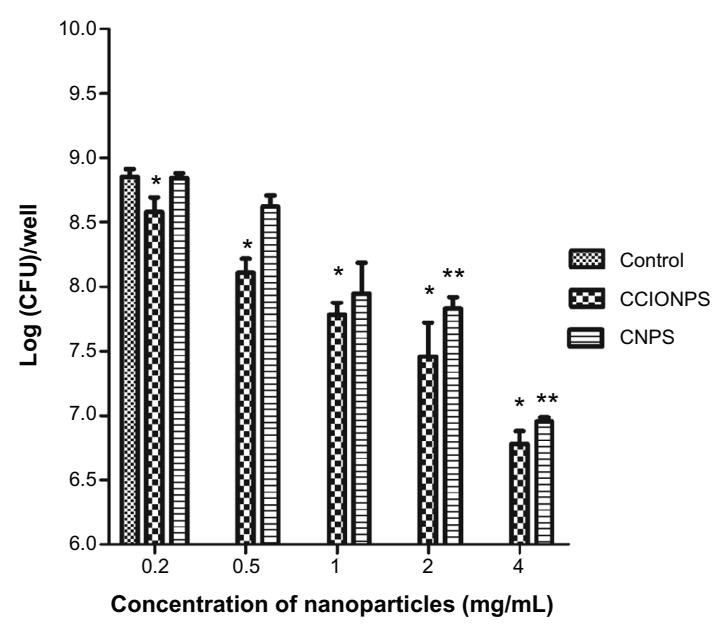

Figure I Effects of different concentrations of nanoparticles on biofilm bacterial growth at after 24 hours of incubation.

Notes: The results are expressed as mean \pm SD $(n=3)$. $* P<0.05$ compared with control sample; **P $<0.05$ compared with control sample (blank).

Abbreviations: CCIONPS, chitosan-coated iron oxide nanoparticles; CFU, colonyforming unit; CNPS, chitosan nanoparticles; SD, standard deviation.

was concentration-dependent. Chitosan nanoparticles also showed restriction at higher concentrations ( 2 and $4 \mathrm{mg} / \mathrm{mL}$ ). Figure 2 reveals that biofilm bacteria increased rapidly in the first 12 hours in the absence of the nanoparticles. This increase was inhibited by the nanoparticles at 6 hours and inhibition was more significant from 6 to 12 hours with decreased biofilm bacteria numbers in the biofilm. These results suggest that chitosan-coated iron oxide nanoparticles are effective in restraining bacterial colonization in biofilm.

\section{Biofilm formation analysis with CV staining}

Biofilm formation presented as relative biomass was calculated and compared to a control sample. CV staining

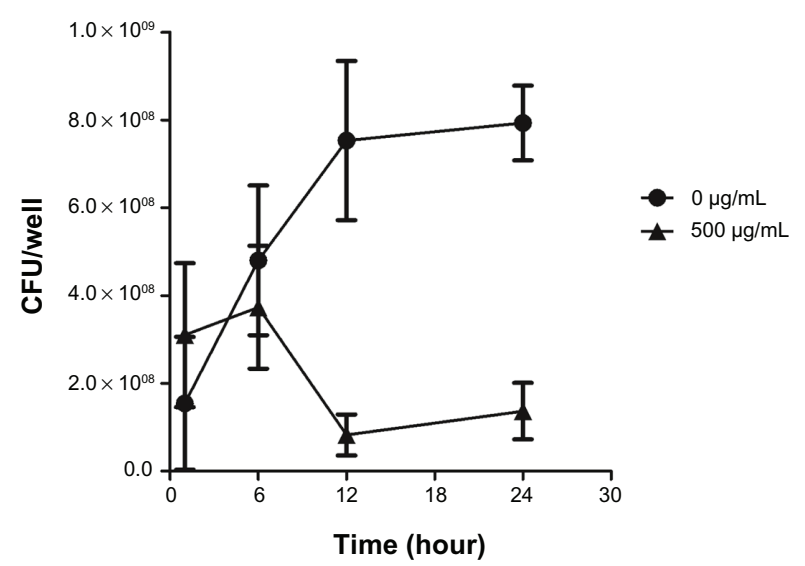

Figure 2 Effects of chitosan-coated iron oxide nanoparticles on biofilm bacterial growth in the first 24 hours at the concentration of $500 \mu \mathrm{g} / \mathrm{mL}$.

Note: The results are expressed as mean \pm SD $(n=3)$.

Abbreviations: CFU, colony-forming unit; SD, standard deviation. measured the reduction of biofilm formation directly on the bottom of the culture plate (Figure 3 ). For all concentrations, biofilm formation was significantly reduced compared to the blank control in the absence of the nanoparticles (53\% reduction in biofilm formation on average). The reduction in biofilm formation was related to the concentration of nanoparticles. There was no significant difference between concentrations of $200 \mu \mathrm{g} / \mathrm{mL}$ and $1 \mathrm{mg} / \mathrm{mL}$. However, there was a significant decrease in biofilm formation at the concentration of $4 \mathrm{mg} / \mathrm{mL}$. In lower concentrations (0.2 and $0.5 \mathrm{mg} / \mathrm{mL}$ ), chitosan-coated iron oxide nanoparticles showed better restriction over chitosan nanoparticles. Figure 4 shows that biofilm formation increased in the first 24 hours in both the chitosan-coated iron oxide nanoparticle treated group $(500 \mu \mathrm{g} / \mathrm{mL})$ and the blank control group. But when incubated with chitosan-coated iron oxide nanoparticles, the biofilm biomass increased at a rate that was much slower than the control.

\section{Biofilm formation by CLSM}

CLSM images revealed a dramatically decreased biofilm formed in the bottom of the culture plate exposed to nanoparticles $(500 \mu \mathrm{g} / \mathrm{mL})$ compared with the control $(0 \mu \mathrm{g} / \mathrm{mL})$. After 2 days of incubation, as shown in three-dimensional imaging, the average biofilm thickness was about $20 \mu \mathrm{m}$ (Figure 5). However, when incubated in the presence of chitosan-coated iron oxide nanoparticles, this thickness decreased to $10 \mu \mathrm{m}$. The viability of the formed biofilm cells was assessed by CLSM using double live/dead staining. The SYTO 9/PI staining visually confirmed a reduced live

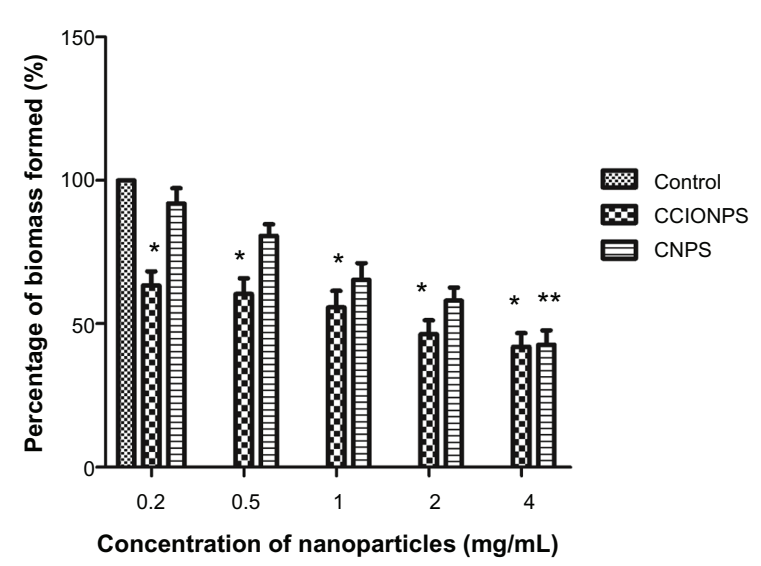

Figure 3 Relative biomass formed after 24 hours of incubation with nanoparticles at different concentrations.

Notes: The results are expressed as mean \pm SD $(n=3)$. *P $<0.05$ compared with control sample; ${ }^{* * P}<0.05$ compared with samples at concentrations of $200 \mu \mathrm{g} / \mathrm{mL}$ and $500 \mu \mathrm{g} / \mathrm{mL}$.

Abbreviations: CCIONPS, chitosan-coated iron oxide nanoparticles; CNPS, chitosan nanoparticles; SD, standard deviation. 


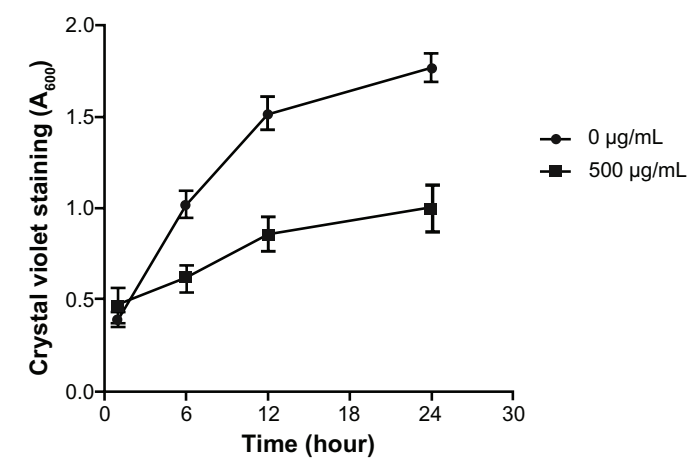

Figure 4 Biomass formed in the first 24 hours of incubation with chitosan-coated iron oxide nanoparticles at a concentration of $500 \mu \mathrm{g} / \mathrm{mL}$.

Note: The results are expressed as mean \pm SD $(n=3)$.

Abbreviation: SD, standard deviation.

bacteria count and indicated significantly higher percentages of dead bacteria (stained red) in the presence of nanoparticles compared to the control (Figure 6). These results are consistent with the biofilm cell count results shown above (Figure 1). CLSM images provided, for the first time, evidence that bacteria death increased and the biofilm thickness decreased in the presence of chitosan-coated iron oxide nanoparticles in this study. These results clearly indicate that the application of chitosan-coated iron oxide nanoparticles inhibited biofilm growth.

\section{Biofilm prevention assay by SEM}

It was observed that the biofilms of the bacteria were fully established on the surface of the polystyrene plates (Figure 7A). Proliferating bacteria covered the whole surface of the material with a large amount of biofilm in the control. The decreased biofilm formation was observed when incubated with chitosan-coated iron oxide nanoparticles at $500 \mu \mathrm{g} / \mathrm{mL}$ (Figure 7B). When the concentration of nanoparticles was increased to $4 \mathrm{mg} / \mathrm{mL}$, only a small protrusion of biofilm was observed (Figure 7C).

\section{Discussion}

As a major pathogen of chronic infection, S. aureus can adhere and form biofilm on medical devices and implants. ${ }^{6}$ S. aureus within biofilms has a better capability of withstanding nutrient deprivation, oxygen radicals, and antibiotics than their planktonic counterpart. ${ }^{25}$ Due to the difficulty of eliminating the biofilm on the surface, biofilm infection attracts much attention in the medical world. ${ }^{26}$ Using nanoparticles to inhibit bacterial growth and biofilm formation is an increasingly attractive approach to both prevention and treatment of infections. As a new approach to treat infection, nanoparticles have many advantages, including high surface-to-volume ratios and nanoscale sizes. ${ }^{27}$ The high surface areas make it easier for the nanoparticle to interact with bacteria. ${ }^{28}$

Chitosan is a natural nontoxic biopolymer with antimicrobial and antifungal activity. ${ }^{16}$ It has a broad spectrum of antimicrobial activity. In Busscher et al's research, ${ }^{29}$ chitosan caused a reduction in bacterial adhesion and was responsible for bacterial death. Biofilm viability after chitosan treatment was lower than that of the control. In previous studies, it was reported that chitosan nanoparticles had been synthesized as antibacterial agents. Qi et al ${ }^{16}$ studied the antibacterial effect of chitosan nanoparticles. Their results showed that chitosan nanoparticles and copper-loaded nanoparticles could inhibit the growth of various bacteria
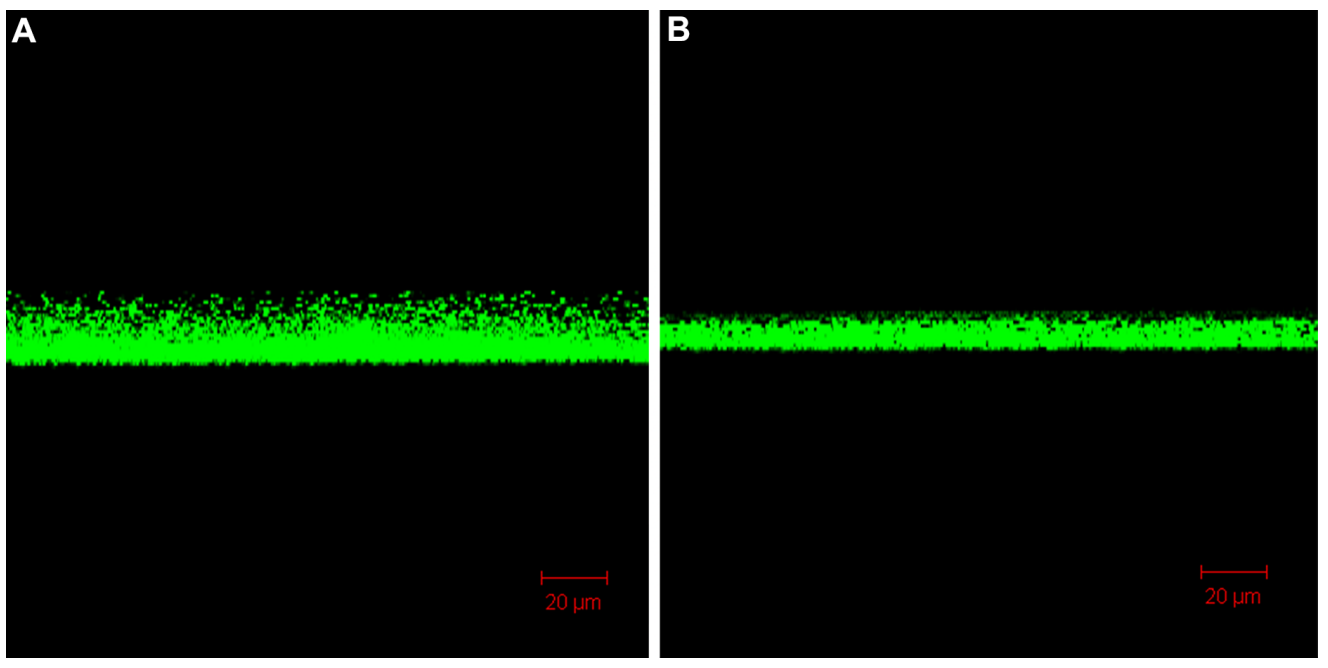

Figure 5 Confocal images show the biofilm thickness after 48 hours of incubation. (A) In the absence of nanoparticles; (B) incubated with chitosan-coated iron oxide nanoparticles at a concentration of $500 \mu \mathrm{g} / \mathrm{mL}$. 

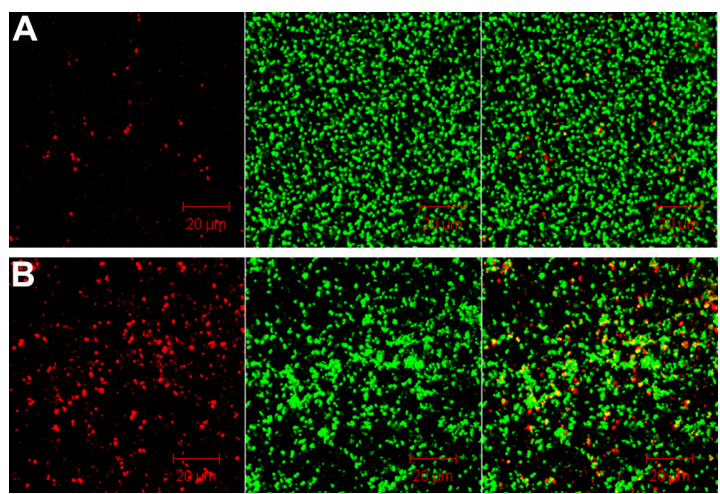

Figure 6 Confocal images show dead bacteria after 48 hours of incubation. (A) In the absence of nanoparticles; (B) incubated with chitosan-coated iron oxide nanoparticles at a concentration of $500 \mu \mathrm{g} / \mathrm{mL}$.

Note: Dead cells (left), living cells (middle), and overlapping images (right).

that they tested. Exposure of $S$. choleraesuis to the chitosan nanoparticles led to the disruption of cell membranes and the leakage of cytoplasm. In our research, chitosan nanoparticles showed biofilm inhibition at high concentrations ( 2 and $4 \mathrm{mg} / \mathrm{mL}$ ). However, in lower concentrations, the inhibition was not obvious compared with chitosan-coated iron oxide nanoparticles. This may be because the size of the chitosan-coated iron oxide nanoparticles $(15-25 \mathrm{~nm})$ was smaller than the chitosan nanoparticles $(200-500 \mathrm{~nm})$. As the concentration of chitosan nanoparticles increased, the inhibition of chitosan nanoparticles became obvious owing to more surface area interacting with bacteria.

There are several mechanisms proposed for the antimicrobial efficiency of chitosan: surface interaction by teichoic acid binding or extraction of membrane lipids. Polycationic charge is known to be an important factor for its antibacterial effect; the charge interaction with bacteria is known to impair the bacterial membrane. ${ }^{30,31}$

The increased surface area owing to the nanosize would further contribute to the chitosan's antibacterial activity. ${ }^{32}$ Inhibiting protein synthesis and restraining microbial growth by forming an external barrier also the antibacterial effect. ${ }^{33}$ Kalishwaralal et al ${ }^{27}$ believe that the inhibitory effect of nanoparticles on the existing biofilm may be due to the presence of water channels throughout the biofilm. Due to the presence of biofilm water channels (pores) for nutrient transportation, nanoparticles may diffuse through the pores and play an antibacterial function in the biofilm.

In our study, the chitosan-coated iron oxide nanoparticles had an antibacterial effect. And the smaller size contributed to their antibacterial effect compared to chitosan nanoparticles. Furthermore, iron oxide may also play a role in antibacterial and anti-biofilm formation. Previous research has shown the antibacterial and anti-biofilm effect of iron oxide nanoparticles like other metal oxide nanoparticles. Taylor and Webster ${ }^{19}$ used iron oxide nanoparticles to study their effect on biofilm formation; the results showed the antibacterial activity of the nanoparticles and prevention of colony assembly, a prerequisite to biofilm formation, was observed after 12 hours. The antibacterial mechanism of metal oxides may involve the generation of hydrogen peroxide or superoxide anion free-radical production. ${ }^{18,34}$ The generation of oxygen radicals can cause DNA strand breaks, inactivate enzymes, and initiate lipid peroxidation. ${ }^{19,35,36}$ The iron oxide nanoparticles binding to cell membranes or cell membrane proteins may also disrupt bacteria functions and lead to bacterial death. ${ }^{17,37,38}$ The biocompatibility of the nanoparticles to human cells increased when the nanoparticles were coated with chitosan. ${ }^{18,20}$ Bare iron oxide nanoparticles have been reported to exert toxic effects. ${ }^{22,39}$ When coated with chitosan, the nanoparticles have a positive surface charge so the adhesion of nanoparticles to negatively charged bacteria increases due to the positively charged surface of the chitosan-coated nanoparticles. ${ }^{19,20,30}$

On the whole, the most desirable advantages of present magnetic nanoparticles over commercial antibiotics are that

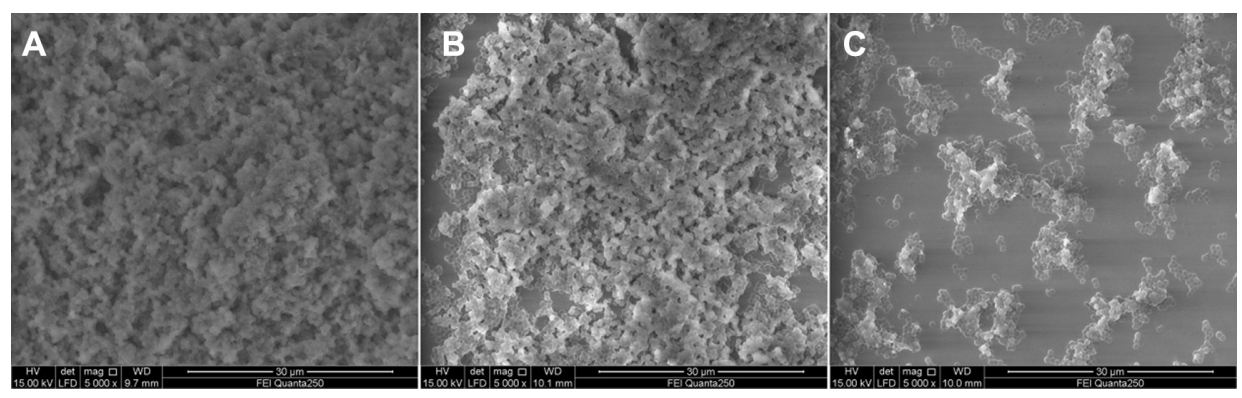

Figure 7 SEM images showing biofilms of the bacteria established on the surface of polystyrene plates. (A) In the absence of nanoparticles; (B) incubated with chitosancoated iron oxide nanoparticles at $500 \mu \mathrm{g} / \mathrm{mL}$; (C) incubated with chitosan-coated iron oxide nanoparticles at $4 \mathrm{mg} / \mathrm{mL}$.

Abbreviation: SEM, scanning electron microscopy. 
they can be magnetically delivered to the local target area and can inhibit biofilm formation. Further research should be performed to study these nanoparticles for anti-infective use.

\section{Conclusion}

In this study, chitosan-coated iron oxide nanoparticles reduced the biofilm formation of $S$. aureus. Results indicated that chitosan-coated iron oxide nanoparticles could decrease the growth of biofilm bacteria, induce biofilm bacteria death, and decrease the biomass formation in the biofilm, which indicates a bright future in biomaterial application.

\section{Author contributions}

All authors contributed toward data analysis, drafting and revising the paper and agree to be accountable for all aspects of the work.

\section{Acknowledgments}

This work was supported by the National Natural Science Foundation of China (No 81401817) and the Jiangsu Natural Science Foundation (No BK20140220). We thank Boyu Liu for creating the figures and correcting the English.

\section{Disclosure}

The authors report no conflicts of interest in this work.

\section{References}

1. Zoubos AB, Galanakos SP, Soucacos PN. Orthopedics and biofilm - what do we know? A review. Med Sci Monit. 2012;18(6):RA89-RA96.

2. Subramani K, Jung RE, Molenberg A, Hammerle CH. Biofilm on dental implants: a review of the literature. Int J Oral Maxillofac Implants. 2009;24(4):616-626.

3. Shi S, Zhang X. Interaction of Staphylococcus aureus with osteoblasts (Review). Exp Ther Med. 2012;3(3):367-370.

4. Urquhart DM, Hanna FS, Brennan SL, et al. Incidence and risk factors for deep surgical site infection after primary total hip arthroplasty: a systematic review. J Arthroplasty. 2010;25(8):1216-1222. e1-e3.

5. Lellouche J, Kahana E, Elias S, Gedanken A, Banin E. Antibiofilm activity of nanosized magnesium fluoride. Biomaterials. 2009;30(30): 5969-5978.

6. Lin MH, Chang FR, Hua MY, Wu YC, Liu ST. Inhibitory effects of 1,2,3,4,6-penta-O-galloyl-beta-D-glucopyranose on biofilm formation by Staphylococcus aureus. Antimicrob Agents Chemother. 2011; 55(3): 1021-1027.

7. Wu J, Xu H, Tang W, Kopelman R, Philbert MA, Xi C. Eradication of bacteria in suspension and biofilms using methylene blue-loaded dynamic nanoplatforms. Antimicrob Agents Chemother. 2009;53(7):3042-3048.

8. Singh R, Ray P, Das A, Sharma M. Penetration of antibiotics through Staphylococcus aureus and Staphylococcus epidermidis biofilms. J Antimicrob Chemother. 2010;65(9):1955-1958.

9. Chaieb K, Kouidhi B, Jrah H, Mahdouani K, Bakhrouf A. Antibacterial activity of Thymoquinone, an active principle of Nigella sativa and its potency to prevent bacterial biofilm formation. BMC Complement Altern Med. 2011;11:29.

10. Hess DJ, Henry-Stanley MJ, Wells CL. Gentamicin promotes Staphylococcus aureus biofilms on silk suture. J Surg Res. 2011;170(2): 302-308.
11. Hoffman LR, D’Argenio DA, MacCoss MJ, Zhang Z, Jones RA, Miller SI. Aminoglycoside antibiotics induce bacterial biofilm formation. Nature. 2005;436(7054):1171-1175.

12. Nishimura S, Tsurumoto T, Yonekura A, Adachi K, Shindo H. Antimicrobial susceptibility of Staphylococcus aureus and Staphylococcus epidermidis biofilms isolated from infected total hip arthroplasty cases. J Orthop Sci. 2006;11(1):46-50.

13. Seil JT, Webster TJ. Reduced Staphylococcus aureus proliferation and biofilm formation on zinc oxide nanoparticle PVC composite surfaces. Acta Biomater. 2011;7(6):2579-2584.

14. Lellouche J, Friedman A, Lahmi R, Gedanken A, Banin E. Antibiofilm surface functionalization of catheters by magnesium fluoride nanoparticles. Int J Nanomedicine. 2012;7:1175-1188.

15. Chen F, Shi Z, Neoh KG, Kang ET. Antioxidant and antibacterial activities of eugenol and carvacrol-grafted chitosan nanoparticles. Biotechnol Bioeng. 2009;104(1):30-39.

16. Qi L, Xu Z, Jiang X, Hu C, Zou X. Preparation and antibacterial activity of chitosan nanoparticles. Carbohydr Res. 2004;339(16): 2693-2700.

17. Chatterjee S, Bandyopadhyay A, Sarkar K. Effect of iron oxide and gold nanoparticles on bacterial growth leading towards biological application. J Nanobiotechnology. 2011;9:34.

18. Inbaraj BS, Kao TH, Tsai TY, Chiu CP, Kumar R, Chen BH. The synthesis and characterization of poly(gamma-glutamic acid)-coated magnetite nanoparticles and their effects on antibacterial activity and cytotoxicity. Nanotechnology. 2011;22(7):075101.

19. Taylor EN, Webster TJ. The use of superparamagnetic nanoparticles for prosthetic biofilm prevention. Int J Nanomedicine. 2009;4: $145-152$.

20. Shi SF, Jia JF, Guo XK, et al. Biocompatibility of chitosan-coated iron oxide nanoparticles with osteoblast cells. Int J Nanomedicine. 2012;7: 5593-5602.

21. Stocks G, Janssen HF. Infection in patients after implantation of an orthopedic device. ASAIO J. 2000;46(6):S41-S46.

22. Mahmoudi M, Sant S, Wang B, Laurent S, Sen T. Superparamagnetic iron oxide nanoparticles (SPIONs): development, surface modification and applications in chemotherapy. Adv Drug Deliv Rev. 2011; 63(1-2):24-46.

23. Choi O, Yu CP, Esteban Fernández G, Hu Z. Interactions of nanosilver with Escherichia coli cells in planktonic and biofilm cultures. Water Res. 2010;44(20):6095-6103.

24. Peng ZX, Tu B, Shen Y, et al. Quaternized chitosan inhibits icaA transcription and biofilm formation by Staphylococcus on a titanium surface. Antimicrob Agents Chemother. 2011;55(2):860-866.

25. Zmantar T, Kouidhi B, Miladi H, Mahdouani K, Bakhrouf A. A microtiter plate assay for Staphylococcus aureus biofilm quantification at various $\mathrm{pH}$ levels and hydrogen peroxide supplementation. New Microbiol. 2010;33(2):137-145.

26. Zodrow KR, Schiffman JD, Elimelech M. Biodegradable polymer (PLGA) coatings featuring cinnamaldehyde and carvacrol mitigate biofilm formation. Langmuir. 2012;28(39):13993-13999.

27. Kalishwaralal K, BarathManiKanth S, Pandian SR, Deepak V, Gurunathan S. Silver nanoparticles impede the biofilm formation by Pseudomonas aeruginosa and Staphylococcus epidermidis. Colloids Surf B Biointerfaces. 2010;79(2):340-344.

28. Bharde AA, Parikh RY, Baidakova M, et al. Bacteria-mediated precursor-dependent biosynthesis of superparamagnetic iron oxide and iron sulfide nanoparticles. Langmuir. 2008;24(11):5787-5794.

29. Busscher HJ, Engels E, Dijkstra RJ, van der Mei HC. Influence of a chitosan on oral bacterial adhesion and growth in vitro. Eur J Oral Sci. 2008;116(5):493-495.

30. Rabea EI, Badawy ME, Stevens CV, Smagghe G, Steurbaut W. Chitosan as antimicrobial agent: applications and mode of action. Biomacromolecules. 2003;4(6):1457-1465.

31. Helander IM, Nurmiaho-Lassila EL, Ahvenainen R, Rhoades J, Roller S. Chitosan disrupts the barrier properties of the outer membrane of gramnegative bacteria. Int J Food Microbiol. 2001;71(2-3):235-244. 
32. Upadya M, Shrestha A, Kishen A. Role of efflux pump inhibitors on the antibiofilm efficacy of calcium hydroxide, chitosan nanoparticles, and light-activated disinfection. J Endod. 2011;37(10):1422-1426.

33. Cobrado L, Azevedo MM, Silva-Dias A, Ramos JP, Pina-Vaz C, Rodrigues AG. Cerium, chitosan and hamamelitannin as novel biofilm inhibitors? J Antimicrob Chemother. 2012;67(5):1159-1162.

34. Yang H, Liu C, Yang D, Zhang H, Xi Z. Comparative study of cytotoxicity, oxidative stress and genotoxicity induced by four typical nanomaterials: the role of particle size, shape and composition. $J$ Appl Toxicol. 2009;29(1):69-78.

35. Rupp F, Haupt M, Klostermann H, et al. Multifunctional nature of UVirradiated nanocrystalline anatase thin films for biomedical applications. Acta Biomater. 2010;6(12):4566-4577.

36. Gordon O, Vig Slenters T, Brunetto PS, et al. Silver coordination polymers for prevention of implant infection: thiol interaction, impact on respiratory chain enzymes, and hydroxyl radical induction. Antimicrob Agents Chemother. 2010;54(10):4208-4218.
37. Park H, Park HJ, Kim JA, et al. Inactivation of Pseudomonas aeruginosa PA01 biofilms by hyperthermia using superparamagnetic nanoparticles. J Microbiol Methods. 2011;84(1):41-45.

38. Subbiahdoss G, Sharifi S, Grijpma DW, et al. Magnetic targeting of surface-modified superparamagnetic iron oxide nanoparticles yields antibacterial efficacy against biofilms of gentamicin-resistant staphylococci. Acta Biomater. 2012;8(6):2047-2055.

39. Mahmoudi M, Simchi A, Imani M, et al. A new approach for the in vitro identification of the cytotoxicity of superparamagnetic iron oxide nanoparticles. Colloids Surf B Biointerfaces. 2010;75(1):300-309.

\section{Publish your work in this journal}

The International Journal of Nanomedicine is an international, peerreviewed journal focusing on the application of nanotechnology in diagnostics, therapeutics, and drug delivery systems throughout the biomedical field. This journal is indexed on PubMed Central, MedLine, CAS, SciSearch ${ }^{\circledR}$, Current Contents ${ }^{\circledR} /$ Clinical Medicine,
Journal Citation Reports/Science Edition, EMBase, Scopus and the Elsevier Bibliographic databases. The manuscript management system is completely online and includes a very quick and fair peer-review system, which is all easy to use. Visit http://www.dovepress.com/ testimonials.php to read real quotes from published authors. 